\title{
Mitochondria as Crucial Players in Demyelinated Axons: Lessons from Neuropathology and Experimental Demyelination
}

\author{
Graham R. Campbell' ${ }^{1}$ and Don J. Mahad', 2,3 \\ ${ }^{1}$ Mitochondrial Research Group, Institute for Ageing and Health, Newcastle University, Framlington Place, Newcastle upon Tyne, \\ NE2 4HH, UK \\ ${ }^{2}$ The Mellen Center for Multiple Sclerosis Treatment and Research, Department of Neurology, Cleveland Clinic Foundation, Cleveland, \\ OH 44195, USA \\ ${ }^{3}$ Department of Neurosciences, The Lerner Research Institute, Cleveland Clinic Foundation, Cleveland, OH 44195, USA
}

Correspondence should be addressed to Don J. Mahad, d.j.mahad@ncl.ac.uk

Received 18 October 2010; Accepted 29 November 2010

Academic Editor: Sreeram Ramagopalan

Copyright ( $) 2011$ G. R. Campbell and D. J. Mahad. This is an open access article distributed under the Creative Commons Attribution License, which permits unrestricted use, distribution, and reproduction in any medium, provided the original work is properly cited.

\begin{abstract}
Mitochondria are the most efficient producers of energy in the form of ATP. Energy demands of axons, placed at relatively great distances from the neuronal cell body, are met by mitochondria, which when functionally compromised, produce reactive oxygen species (ROS) in excess. Axons are made metabolically efficient by myelination, which enables saltatory conduction. The importance of mitochondria for maintaining the structural integrity of myelinated axons is illustrated by neuroaxonal degeneration in primary mitochondrial disorders. When demyelinated, the compartmentalisation of ion channels along axons is disrupted. The redistribution of electrogenic machinery is thought to increase the energy demand of demyelinated axons. We review related studies that focus on mitochondria within unmyelinated, demyelinated and dysmyelinated axons in the central nervous system. Based on neuropathological observations we propose the increase in mitochondrial presence within demyelinated axons as an adaptive process to the increased energy need. An increased presence of mitochondria would also increase the capacity to produce deleterious agents such as ROS when functionally compromised. Given the lack of direct evidence of a beneficial or harmful effect of mitochondrial changes, the precise role of increased mitochondrial presence within axons due to demyelination needs to be further explored in experimental demyelination in-vivo and in-vitro.
\end{abstract}

\section{Introduction}

Axons are unique structures in the central nervous system with much of their cytoplasm found at great distances from the neuronal cell body. Myelination of axons is essential for fast conduction of action potentials and their metabolic efficiency [1]. Axonal degeneration in demyelinating diseases such as multiple sclerosis (MS) indicates the importance of trophic support that myelin lends to axons whilst also providing protection from various extracellular insults. Mitochondria, the ubiquitous energy-producing organelles, are found within axons. They are vastly dynamic and locate to areas in which they are most needed and, as we will discuss in this review, appear highly adaptable to subtle energy changes within the axon. We review mitochondrial changes that follow demyelination and indicate the damaging consequences of mitochondrial failure for axons.

\section{Mitochondria}

Mitochondria are charged with supplying the vast amount of ATP required in eukaryotic cells. Other important roles in calcium buffering and apoptosis cannot be underestimated [2]. The respiratory chain which is responsible for the process of oxidative phosphorylation, ultimately resulting in the production of ATP from ADP, consists of complex Icomplex IV and an additional ATP synthase.

Mitochondria contain the only extranuclear DNA in the cell (mtDNA). The mitochondrial genome holds 13 protein encoding genes which incorporate into complex I, 
complex III, complex IV, and ATP synthase. Complex II is the only complex with all subunits encoded by nuclear DNA, which, from an investigative point of view, is highly advantageous $[3,4]$. MtDNA mutation (point mutations and deletions) led energy deficiency states frequently affect the central nervous system (CNS) in patients with primary mitochondrial diseases.

\section{A Question of Energy for Axons}

The vast energy requirement of axons is highlighted by the location of the sodium potassium ATPase $\left(\mathrm{Na}^{+} / \mathrm{K}^{+}\right.$ATPase) which extends along myelinated segments of the axons (internodes) [5]. The $\mathrm{Na}^{+} / \mathrm{K}^{+}$ATPase facilitates the rapid exchange of sodium for extracellular potassium, through the process of active transport and is thus a major consumer of energy in the CNS [6]. Sodium channels play an important role in axons, and their clustering at the nodes of Ranvier facilitate the fast propagation of action potentials or "saltatory conduction" and allow influx of sodium into the axon. Different isoforms exist, but the accepted forms known to exist in neurons are $\mathrm{Na}_{\mathrm{v}} 1.1, \mathrm{Na}_{\mathrm{v}} 1.2$, and $\mathrm{Na}_{\mathrm{v}}$ 1.6. Those expressed on axonal membranes are predominantly $\mathrm{Na}_{\mathrm{v}} 1.2$ and $\mathrm{Na}_{\mathrm{v}} 1.6$ [7]. The importance of the persistent sodium influx allowed by $\mathrm{Na}_{\mathrm{v}} 1.6$ is highlighted by the redistribution of the channel along nonmyelinated axons to maintain action potentials [8]. During development in the premyelination state, $\mathrm{Na}_{\mathrm{v}} 1.2$ channels support action potentials [9] which are soon replaced following myelination with $\mathrm{Na}_{\mathrm{v}} 1.6$ channels, identified to allow a persistent current of sodium. Myelin also induces the clustering of sodium channels at nodes of Ranvier [10]. In the CNS, mitochondria were presumed to reside in the nonmyelinated segments, nodes of Ranvier, based on findings in the peripheral nervous system (PNS); however, recent evidence suggests that mitochondria preferentially locate in the internodes [11], at least in small diameter axons, which would fit with the energy demand hypothesis. Evidence for the need of precise location of mitochondria within axons is observed in growth cones. An elegant study by Morris and Hollenbeck in 1993 showed that the presence of mitochondria in neurons is coordinated with axonal outgrowth. They showed that by blocking the growth of a number of axons and then visualising the mitochondrial content, the preferential location of the mitochondria was in the outgrowing axons, particularly in the terminal ends [12]. Whilst ATP can readily diffuse into the cytosol, it appears that the precise location of mitochondria is important.

Axons, both myelinated and unmyelinated, are an excellent forum to understand the relationship between mitochondria and the differing energy demands of the CNS, given the difference in ion channel expression in these axons [13]. The lamina cribrosa is a region of the optic nerve that is unmyelinated with myelination of fibres occurring at the posterior border. Complex IV activity was assessed in fibres of the lamina cribrosa [3]. Complex IV consumes $90 \%$ of cellular oxygen [2] and is involved in proton pumping across the inner mitochondrial membrane. Perhaps, importantly, numerous inhibitors of complex IV exist including nitric oxide, a competitive inhibitor [14], whilst its more toxic derivative, peroxynitrite, can irreversibly inhibit both complex I and complex IV [15]. Complex IV activity was found to be vastly increased in the unmyelinated segment compared to the myelinated segment which corresponded with an increase in complex IV subunit II protein level $[16,17]$. The distribution of complex IV activity correlated with certain isoforms of sodium channels. Both $\mathrm{Na}_{\mathrm{v}} 1.1$ and $\mathrm{Na}_{\mathrm{v}} 1.6$ were found to increase in intensity with lack of myelin whilst there was little predilection for $\mathrm{Na}_{\mathrm{v}} 1.2$ in either region [18]. These findings in control subjects suggest a physiological role for the increased presence of mitochondria in the unmyelinated segments compared with myelinated segments of optic nerve axons.

\section{Loss of Compartmentalisation of Electrogenic Machinery and a Question of Energy for Demyelinated and Dysmyelinated Axons}

Several groups have predicted metabolic changes within axons based on changes in ion channel distribution following demyelination and dysmyelination (Figure 1) [19-21]. There has been a number of studies with animal models in which genes for myelin proteins have been knocked out or toxic insults to myelin have been introduced.

In a mouse model of hypomyelination caused by gene knockout encoding the myelin basic protein gene, complex IV activity was found to be increased associated with an increase in mitochondrial density suggestive of adaptive changes to this change in myelin [20]. Furthermore, in a proteolipid protein (PLP) overexpressing mouse model, dysmyelination at 1 month followed by complete demyelination at 4 months was associated with an increase in mitochondrial density and complex IV activity compared to wild type [19]. Axonal degeneration was not evident at this time point. In a hemizygous PLP overexpressing mouse model predisposing to only partial demyelination, whilst mitochondrial density increased, complex IV activity remained similar to wild type. Electron microscopy revealed the presence of degenerating axons suggesting a lack of compensation from complex IV predisposed to the phenotype. These animal models show the relationship between disruptions to myelin and changing energy demands compensated for by alterations in mitochondria.

In one particular model in which antiserum to galactocerebroside was used to selectively demyelinate cat optic nerve, a significant increase in mitochondrial number was observed which reached a peak at seven days after injection [21]. The authors suggest that this is an adaptive feature of the demyelinated axons particularly as this coincided with a change from conduction block to slowed conduction [22]. In a demyelination model induced by Theiler's murine encephalomyelitis virus (TMEV), neurological dysfunction results from progressive nervous system demyelination. Mitochondrial numbers were found to be significantly increased in demyelinated axons compared to controls [23]. Interestingly, mitochondrial numbers correlated well 


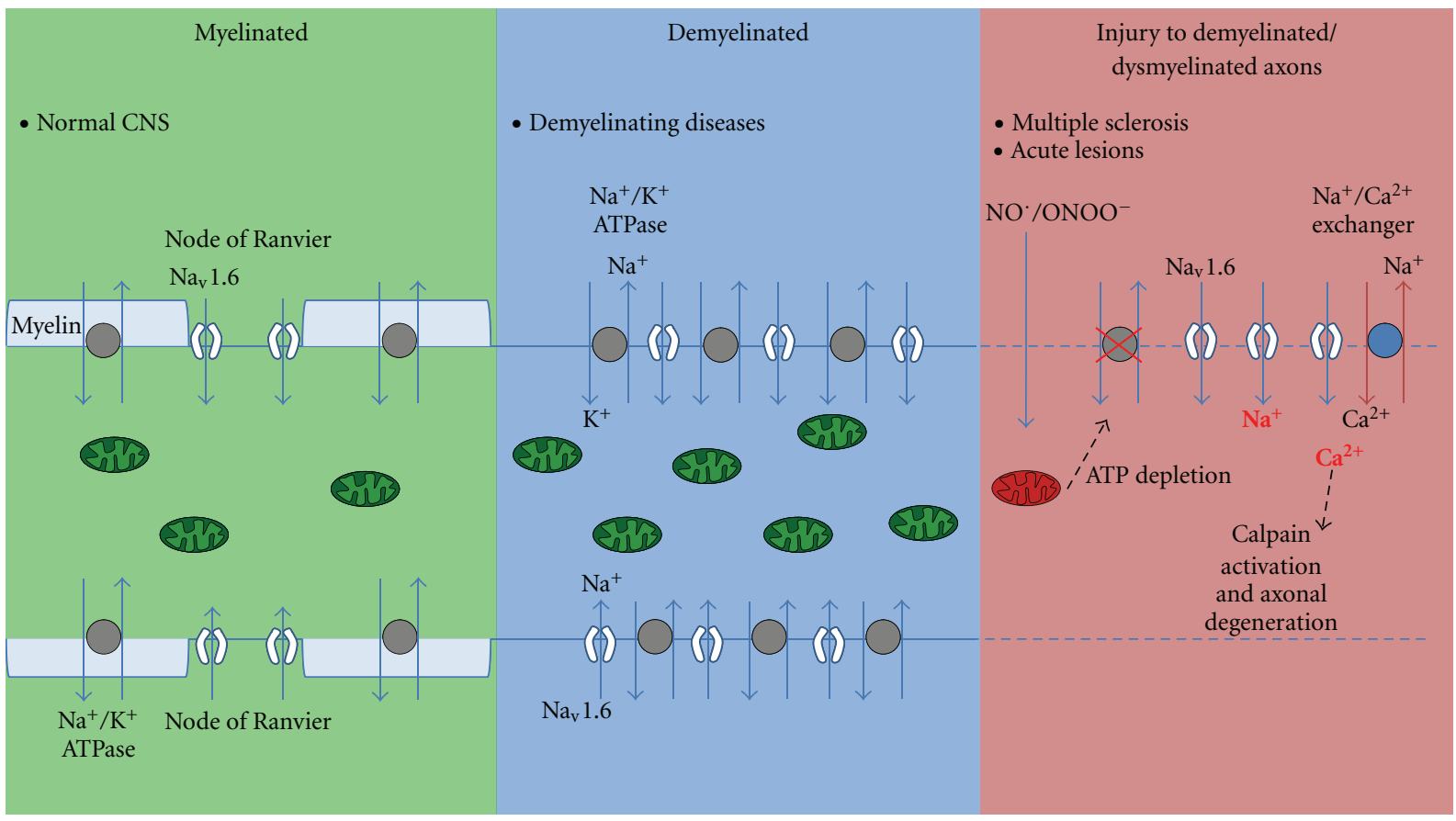

FIGURE 1: Mitochondrial changes within axons in response to demyelination and further cytoskeletal injury. Sodium channels $\left(\mathrm{Na}_{\mathrm{v}} 1.6\right)$ are located at nodes of Ranvier in the CNS whilst the $\mathrm{Na}^{+} / \mathrm{K}^{+}$ATPase extends the myelinated segments (internodes). To facilitate the extended energy demand of the axon, mitochondria have been shown to distribute throughout the axonal cytoplasm in small axons in the CNS (green). The redistribution of sodium channels facilitates the continuation of action potentials along the demyelinated axons (blue). Mitochondrial dysfunction can leave the axon vulnerable as observed in acute and chronic stages of MS (red). It is hypothesised that the failure of the $\mathrm{Na}^{+} / \mathrm{K}^{+}$ATPase, possibly due to mitochondrial dysfunction, can lead to increased sodium concentrations in the axoplasm. Reversal of the $\mathrm{Na}^{+} / \mathrm{Ca}^{2+}$ exchanger can ensue, resulting in toxic calcium levels and downstream processes with consequent axonal degeneration. Mitochondrial dysfunction (red) possibly by inflammatory products such as nitric oxide $(\mathrm{NO})$ and peroxynitrite $\left(\mathrm{ONOO}^{-}\right)$ can lead to membrane depolarisation and retrograde transport toward the neuronal soma (arrow to the left). A population of axons have been shown to exist without the $\mathrm{Na}^{+} / \mathrm{K}^{+}$ATPase which would also have a similar effect to an energy defect.

with the percentage of total spinal cord demyelination and strong correlations were found between the number of degenerating axons and intra-axonal mitochondria in demyelinated axons, raising the possibility that increases of intra-axonal mitochondria may be detrimental to axons. Mitochondrial density was still increased after 6 months. Our view, partly supported by observations in unmyelinated axons, is that an increase in mitochondria per se, as long as the function is not compromised, is not damaging to the axons. However, mitochondrial defects in situations of elevated energy needs, as seen in some demyelinated axons, may be even more detrimental due to the toxic effects of dysfunctional mitochondria.

Mitochondrial defects in experimental demyelinating models implicate the organelle in the pathogenesis of axonal degeneration. Post-translational modification of mitochondrial respiratory chain subunits as well as transcriptionally regulated changes in mitochondrial function have been observed in animal models of MS [24-27]. Moreover, the addition of reactive oxygen species scavengers resulting in decreased optic nerve degeneration suggests a role for mitochondrial injury via oxidative stress and identifies mitochondrially targeted agents as potential therapy in MS [28].

\section{The Importance of Mitochondrial Transport, Fusion, Fission, and Biogenesis for Demyelinated Axons}

The observation of an increased mitochondrial presence within demyelinated axons raises a number of important questions including how the mitochondrial changes occur. The possible explanations include an increase in anterograde transport, decrease in retrograde transport, increase in fusion, decrease in fission, and mitochondrial biogenesis within axons.

Neurons have developed a highly sophisticated transport system to meet the needs of the axon with machinery for both transport of proteins away from the cell body, into the far reaches of the axon, anterograde transport, and a system to transport aberrant proteins back to the cell body for degradation, retrograde transport. Two pools of mitochondria exist in axons, those that are stationary, which comprise $70 \%$ of axonal mitochondria, and those that are mobile. Mobile mitochondria have been found to move on both microtubules and actin-microfilaments within the axon. A 1995 study used toxic agents which were applied to axons that disrupted either microtubules or actin-microfilament [29]. The authors concluded that 
mitochondrial velocity within axons is determined by coordination of microtubule and actin-microfilament based movements.

There are two motor transport systems responsible for anterograde and retrograde movements of mitochondria, kinesins and dyneins, respectively. Kinesin motors are responsible for anterograde transport whilst dynein motors are responsible for retrograde transport along microtubules. Kinesins of the kinesin-1 family associate with mitochondria as well as vesicles containing, among other proteins, amyloid precursor protein (APP). Sites of APP accumulation in postmortem tissues are readily used to detect disruption of fast axonal movement $[30,31]$. Inhibition of kinesin-1 in Drosophila melanogaster has been shown to inhibit mitochondrial transport [32]. The importance of anterograde mitochondrial transport is highlighted by mutations of one member of the kinesin family, Kif1 $\beta$, shown to transport mitochondria. These mutations can lead to the axonal form of Charcot-Marie-Tooth disease (CMT2a). Heterozygous knockout mice showed symptoms similar to CMT2a with progressive muscle weakness. The ATPase activity of the motor was found to be reduced, thus, suggestive of a functional loss of motor activity [33]. Retrograde movement of microtubules appears to require dynein motors [34], although it is known that several kinesin motor families have retrograde movement, however, the velocity at which these move indicates they are unlikely to be involved in axonal mitochondrial transport. Myosin motors are implicated on actin-microfilaments due to the bidirectional movement of mitochondria. Myosin V is a likely candidate as it has been shown to move organelles at similar rates as observed with mitochondria in axons [29].

It is clear from recent studies that retrograde movement of mitochondria occurs in situations of mitochondrial dysfunction. For instance in familial amyotrophic lateral sclerosis where mutations in the antioxidant enzyme $\mathrm{Cu} / \mathrm{Zn}$ superoxide dismutase-1 (SOD1) which accounts for $20 \%$ of all familial cases, it has been shown that this mutation inhibits anterograde transport but not retrograde transport [35]. Furthermore, when the complex III inhibitor, antimycin, was used in a cell culture model of neurons derived from dorsal root ganglion, the number of mitochondria associated with retrograde transport significantly increased [36].

There was understood to be a mitochondrial docking protein for the stationary pool in axons although the actual identity of this protein remained unknown for some years. In a recent study, syntaphilin, a neuron-specific protein was identified as this docking protein [37]. The authors show that mitochondria and syntaphilin colocalise within axons, syntaphilin immobilises the organelle, and in syntaphilin knockout mice mitochondrial motility increased but their number significantly reduced in axons.

Mitochondria are far more dynamic organelles than depicted in textbook images, existing in networks rather than in isolation. Large networks of mitochondria can form through the process of fusion and can be broken up through the action of fission. Mitochondrial fusion machinery consists of the mitofusins MTF1 and MTF2 as well as the GTPase OPA1, responsible for both outer and inner membrane fusion, respectively [38]. DRP1 is the key regulator of mitochondrial fission [39]. The importance of these processes can be observed again in CMT2a which is associated with a number of mutations in MFN2 [40]. Similarly, mice that lack MFN1, MFN2, or OPA1, all responsible for mitochondrial fusion, do not survive beyond midgestation [40-42]. OPA1 mutations in humans cause autosomal dominant optic atrophy (ADOA) characterised by the degeneration of retinal ganglion cells and the optic nerve axons [43].

It was long assumed that axons lack the protein synthesis machinery required for organelle and protein biogenesis and thus the neuronal cell body would operate as this site, probably close to the nucleus, followed by distribution of the organelle to the axons [44]. However, recent evidence suggests that local protein synthesis can occur in axons [45]. Newly synthesised mtDNA has been shown to be present not only in the cell body but throughout axons. The authors also show that Drp1, an essential mitochondrial fission protein, is present in axonal mitochondria. It is probable that newly synthesised mitochondria, supported by an increase in anterograde transport, fuse with already immobile mitochondria at specific energy-demanding sites. How important each of the above processes are for the mitochondrial changes following demyelination needs to be further investigated.

\section{Mitochondrial Changes in Multiple Sclerosis}

MS is the most common demyelinating disease of the central nervous system and, as such, much can be learnt from studies of the demyelinated axons [46]. Demyelination in MS classically occurs with inflammation and is associated with axonal loss which underlies neurological impairment in MS [47]. Demyelinated lesions associated with various degrees of inflammation, represent the pathological hallmarks of the disease which have been studied since the 19th century. Axonal degeneration is most prevalent in active lesions where inflammation is greatest. In chronic lesions, many surviving axons remain chronically demyelinating with only a few undergoing degeneration at any given time point. Axonal injury is highlighted by dephosphorylation of neurofilament side arms and has been observed in models of de/dysmyelination [48] and in acute and chronic MS lesions [49]. In addition, accumulation of beta-amyloid precursor protein ( $\beta$-APP) has been shown to be a marker for axonal damage in cases of diffuse axonal injury [50]. The protein is known to be transported by fast axonal transport which is disrupted in axonal injury leading to accumulation of organelles and intra-axonal proteins around the site of injury [51]. It is only recently that mitochondrial changes have been studied in these axons.

Mitochondrial activity in the form of complex IV has been shown to be increased in inactive areas of chronic lesions associated with an increase of mitochondrial mass (Figure 1) [52, 53]. The increase in complex IV has been noted using several biochemical techniques [54]. Besides the activity and density, the mobility of mitochondria within 


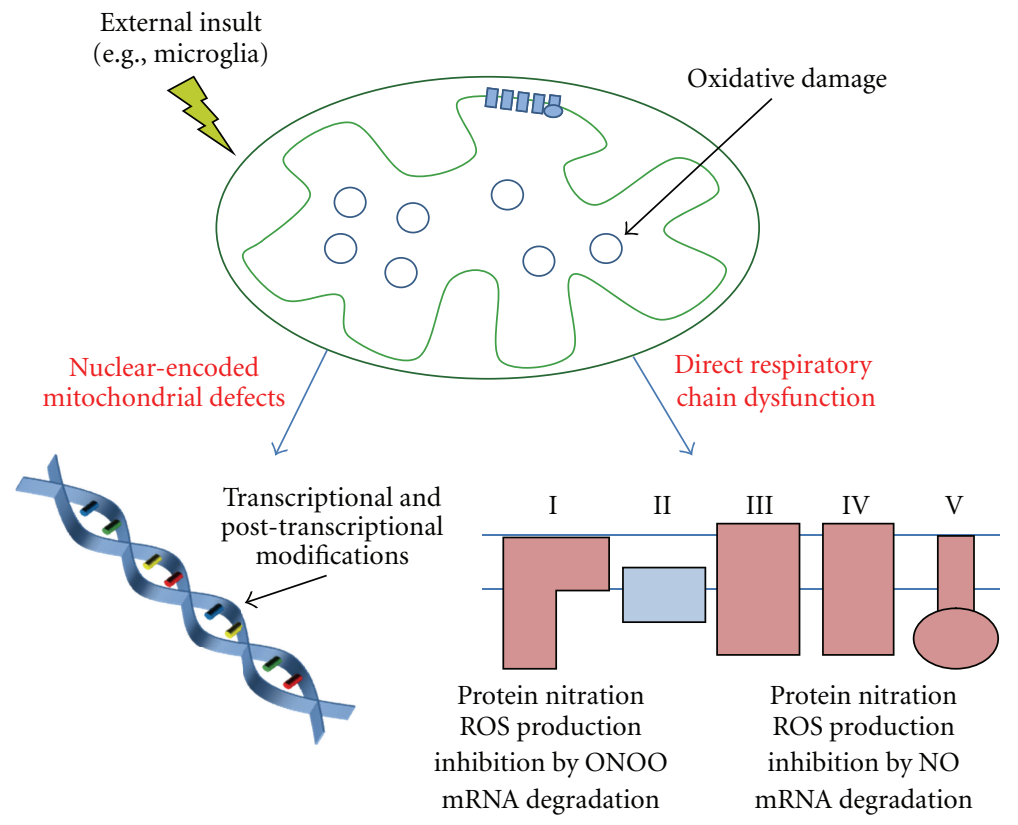

FIGURE 2: Mitochondrial dysfunction in MS and its models. Inflammation and the resulting toxic environment can have multiple effects on mitochondria including mtDNA damage (left arrow) and respiratory chain modifications (right arrow). Increased mtDNA copy number and mtDNA deletions have been observed in MS, which may be a related phenomenon. Actual respiratory chain defects are observed where all but complex II (blue) have both nuclear and mitochondrial DNA-encoded subunits. Defects include protein nitration affecting complexes I and IV observed in EAE, reduction in complex I activity in chronic lesions with reductions in complex I and III in nonlesional motor cortex. Complex IV activity is increased, along with mitochondrial mass, in chronic axons within nonpathological axons whilst a reduction is observed in pathological axons. The recognised production of reactive oxygen species particularly stemming from complex I and III, along with complex inhibition by nitric oxide and peroxynitrite, a result of chronic inflammation, points to the high vulnerability of mitochondria in MS.

axons may also be influenced by demyelination. The expression of axon-specific mitochondrial docking protein, syntaphilin, in chronic lesions indicates a potentially immobile reservoir that supplies the necessary energy in demyelinated axons [52]. These axons were morphologically intact, except for demyelination, and did not show an accumulation of $\beta$ APP. The fact that the above mitochondrial changes were observed in approximately $50 \%$ of morphologically intact axons that were phosphorylated provides support for the adaptive or compensatory theory.

Complex IV defects have been noted in both nonphosphorylated and APP-positive chronically demyelinated axons with an associated decrease of mitochondrial mass in the former but not latter case, suggesting differing mechanisms (Figure 2) [52]. Another study finds agreement with the reduction in ATP synthase expression in MS lesions [55]. Enhanced immunoreactivity of the mitochondrial heat shock protein (mtHSP70) in chronic lesions has been noted indicating an environment of oxidative stress $[53,56]$.

Although not directly located to axons, other mitochondrial defects have been shown in MS tissue including complex I dysfunction in white matter lesions [54] as well as reduced complex I and complex III dysfunction in nonlesional motor cortex [57]. This study based on gene expression profiles, revealed a decrease in nuclear encoded subunits of complex I, complex III, complex IV, and ATP synthase (Figure 2). Whilst this was reflected in the reduced activities of complex I and complex III whilst complex IV activity remained the same suggesting a compensatory mechanism. Furthermore, in white matter MS lesions, oxidative damage to mtDNA has been reported [54]. The compensatory mitochondrial changes in chronic lesions appear to be an adaptive process to the demyelinationinduced energy changes that we have discussed previously. The increase in complex IV, which has been shown in a number of studies, may also result from a reduction in other complex activities, though whether this is enough to sustain ATP production over a period of time is unknown. The adaptive changes of mitochondria in demyelinated axons, however, will increase the vulnerability of these axons to further energy defects. The mitochondrial defects are likely to compromise the function and structure of the surviving demyelinated axons in MS, contributing to the neurological disability and its progression.

\section{Mechanisms of Axonal Degeneration Resulting from Demyelination}

MS is viewed as a disease of two stages with vast axonal loss in the acute stage, associated with inflammation [30, 49]. Direct T-cell cytotoxicity, matrix metalloproteins, and cytokines have all been implicated in axonal transaction [58-60]. Incomplete and failure of remyelination results in gradual slow-burning loss of chronically demyelinated 
axons which significantly contributes to global axonal loss [61], a mechanism that has also been observed in the normal-appearing white matter [62]. In the chronic stage of disease, a cumulative loss of axons over many years is observed despite a decrease in inflammatory activity. Thus, it is understood that this represents a slow-burning axonal disruption phenomenon to the point where the CNS cannot compensate. The exact mechanism through which chronically demyelinated axons degenerate is not known.

There is no doubt that sodium channels have an important role to play, both in restoring conduction in demyelinated axons and in their degeneration (Figure 2). It is hypothesised that the accumulation of sodium in the axon can lead to reversal of the sodium-calcium exchanger resulting in potentially lethal calcium levels [63]. Evidence for this initial mechanism is observed in postmortem tissue of acute MS lesions where APP positive axons are associated with co-localisation of $\mathrm{Na}_{\mathrm{v}} 1.6$ channels and the $\mathrm{Na}^{+} / \mathrm{Ca}^{2+}$ exchanger [64]. Elevated calcium levels are further exacerbated by an increase in expression of N-type voltage-gated calcium channels in MS [65]. Calcium can activate multiple pathways including those of calpains of which high levels are recognised to be a final common pathway to cell death $[13,66]$. Calpain inhibitors, preventing axonal loss, identify the calcium-mediated pathway as the final step toward axonal degradation [67]. Evidence for the importance of sodium channels in axonal degeneration stems from a number of in vivo studies which show axonal protection as a result of the use of sodium channel blockers [68-71]. Loss of axons was ameliorated in the corticospinal tract of EAE animals when phenytoin treatment was given [70] which coincided with improving clinical outcomes. A protection against reduced conduction velocity was also observed. Flecainide and lamotrigine have also shown this effect in the same model with an improved functional outcome and reduction in neurological deficit, respectively, $[68,69]$. It has been noted that exacerbation of EAE followed the withdrawal of two sodium channel blockers, phenytoin and carbamazepine [72] which was probably due to the associated marked increase in inflammatory cells.

A recent clinical trial of lamotrigine in secondary progressive MS patients produced intriguing results [73]. The primary outcome measure, white matter volume, and whole brain volume, showed an unexpected loss of partial cerebral volume in the treatment group. This difference between treated group and controls was no longer apparent once lamotrigine was discontinued, the whole brain volume increased in the treated group. As sodium channels are expressed in other cell types including immune cells, the outcome measurement of brain volume may be effected by oedema and loss of inflammatory cells as well as direct effects on neurodegeneration. For instance, the administration of phenytoin decreases the number of inflammatory cells by $75 \%$ [74], which may explain brain volume decreases in the treatment group.

Complementary to the hypothesis of increased axonal sodium via persistent sodium entry through $\mathrm{Na}_{\mathrm{v}} 1.6$, is that of the lack of the sodium-potassium ATPase on some chronically demyelinated axons. This is not a rare phenomenon with more than half of chronic lesions containing axons in which only $50 \%$ express the $\mathrm{Na}^{+} / \mathrm{K}^{+}$ATPase [5]. In these axons, already high sodium axonal levels are exacerbated by the failure to extrude sodium via the $\mathrm{Na}^{+} / \mathrm{K}^{+}$ATPase [75]. The fact that these axons exist at all in chronic lesions is interesting. One of the reasons may be the lack of $\mathrm{Na}_{\mathrm{v}} 1.6$ channels along the axolemma, as only a third of axons in chronic lesions express $\mathrm{Na}_{\mathrm{v}} 1.6$ and do so only in a patchy rather than continuous pattern. These axons do not appear to be degenerating, highlighted by the lack of positive APP staining. Further evidence comes from the lack of association with the sodium-calcium exchanger with these axons[76]. It is probable that rather than suffering from degeneration, they lack the capacity to electrically conduct as the $\mathrm{Na}^{+} / \mathrm{K}^{+}$ATPase is responsible for returning the axonal membrane potential to normal following an action potential.

Mitochondrial adaptations in demyelinated axons appear crucial to maintaining the axonal integrity and to preventing oxidative damage [52]. This is a point often missed but may turn out to be just as important as an increase in energy demand. In those axons with cytoskeletal changes induced by demyelination mitochondrial function is of high importance in order to facilitate the operation of the sodiumpotassium ATPase. An energy-deficient state mediated by any mode of mitochondria dysfunction will render the axon nonconductive. Indeed nitric oxide, an inhibitor of complex IV, has been shown to contribute to axonal dysfunction and degeneration $[55,77]$.

Mitochondrial changes may have an underlying yet under appreciated role in MS and may explain the lack of consistent relationship between inflammation, demyelination, and axonal loss. Indeed, conduction block rather than axonal loss has been shown to occur following demyelination [78]. Energy deficiency or mitochondrial defects may cause conduction block particularly in demyelinated axons [79]. The preferential loss of small-diameter axons in MS with relative preservation of large axons may also be explained by these mitochondrial changes. The relatively reduced volume-to-surface-area ratio in small axons may indicate that small-diameter axons do not have the same capacity for mitochondrial changes as their large axon counterparts.

\section{Conclusions}

Mitochondria's multiple functions implicate them as crucial players in a healthy central nervous system, and their functional failure, therefore, can result in catastrophic events, as evident in primary mitochondrial disorders. Energy changes associated with demyelination and dysmyelination including the redistribution of ion channels and pumps requires an associated increase in mitochondria. Neuropathological studies and evidence from animal models suggest that demyelinated axons are heavily reliant on mitochondria to fulfil both energy demands and oxidative stress protection. Indirect observations point to the fact that this is an essential requirement of the axon rather than a pathogenic event. An energy deficit is likely to lead to an accumulation of sodium in axoplasm, reversal of sodium/calcium exchange, 
and axonal degeneration. An energy deficit may be the result of not just oxidative phosphorylation defects but also altered mitochondrial transport, fission, fusion, and biogenesis. Given the limitations of sodium channel blockade and the important role of mitochondria in CNS disorders with demyelination, protection of axonal mitochondria seems a priority to preserve demyelinated axons, and developing treatments warrants further investigation.

\section{Acknowledgments}

The authors would like to thank the Wellcome Trust, Multiple Sclerosis Society (UK), Newcastle Healthcare Charity, and Newcastle upon Tyne Hospitals NHS Charity for funding their work.

\section{References}

[1] X. Yin, R. C. Baek, D. A. Kirschner et al., "Evolution of a neuroprotective function of central nervous system myelin," Journal of Cell Biology, vol. 172, no. 3, pp. 469-479, 2006.

[2] S. DiMauro and E. A. Schon, "Mitochondrial respiratorychain diseases," The New England Journal of Medicine, vol. 348, no. 26, pp. 2656-2668, 2003.

[3] R. W. Taylor and D. M. Turnbull, "Mitochondrial DNA mutations in human disease," Nature Reviews Genetics, vol. 6, no. 5, pp. 389-402, 2005.

[4] R. W. Taylor, A. M. Schaefer, M. J. Barron, R. McFarland, and D. M. Turnbull, "The diagnosis of mitochondrial muscle disease," Neuromuscular Disorders, vol. 14, no. 4, pp. 237-245, 2004.

[5] E. A. Young, C. D. Fowler, G. J. Kidd et al., "Imaging correlates of decreased axonal $\mathrm{Na}^{+} / \mathrm{K}^{+}$ATPase in chronic multiple sclerosis lesions," Annals of Neurology, vol. 63, no. 4, pp. 428435, 2008 .

[6] S. G. Waxman, "Mechanisms of Disease: sodium channels and neuroprotection in multiple sclerosis-current status," Nature Clinical Practice Neurology, vol. 4, no. 3, pp. 159-169, 2008.

[7] M. R. Kaplan, M. H. Cho, E. M. Ullian, L. L. Isom, S. R. Levinson, and B. A. Barres, "Differential control of clustering of the sodium channels $\mathrm{Na}_{\mathrm{v}} 1.2$ and $\mathrm{Na}_{\mathrm{v}} 1.6$ at developing CNS nodes of Ranvier," Neuron, vol. 30, no. 1, pp. 105-119, 2001.

[8] J. A. Black, M. Renganathan, and S. G. Waxman, "Sodium channel $\mathrm{Na}_{\mathrm{v}} 1.6$ is expressed along nonmyelinated axons and it contributes to conduction," Molecular Brain Research, vol. 105, no. 1-2, pp. 19-28, 2002.

[9] S. G. Waxman, J. A. Black, J. D. Kocsis, and J. M. Ritchie, "Low density of sodium channels supports action potential conduction in axons of neonatal rat optic nerve," Proceedings of the National Academy of Sciences of the United States of America, vol. 86, no. 4, pp. 1406-1410, 1989.

[10] P. Shrager, "Sodium channels in single demyelinated mammalian axons," Brain Research, vol. 483, no. 1, pp. 149-154, 1989.

[11] J. M. Edgar, M. C. McCulloch, C. E. Thomson, and I. R. Griffiths, "Distribution of mitochondria along smalldiameter myelinated central nervous system axons," Journal of Neuroscience Research, vol. 86, no. 10, pp. 2250-2257, 2008.

[12] R. L. Morris and P. J. Hollenbeck, "The regulation of bidirectional mitochondrial transport is coordinated with axonal outgrowth," Journal of Cell Science, vol. 104, no. 3, pp. 917927, 1993.
[13] S. G. Waxman, "Axonal conduction and injury in multiple sclerosis: the role of sodium channels," Nature Reviews Neuroscience, vol. 7, no. 12, pp. 932-941, 2006.

[14] G. C. Brown and C. E. Cooper, "Nanomolar concentrations of nitric oxide reversibly inhibit synaptosomal respiration by competing with oxygen at cytochrome oxidase," FEBS Letters, vol. 356, no. 2-3, pp. 295-298, 1994.

[15] E. Clementi, G. C. Brown, M. Feelisch, and S. Moncada, "Persistent inhibition of cell respiration by nitric oxide: crucial role of S-nitrosylation of mitochondrial complex I and protective action of glutathione," Proceedings of the National Academy of Sciences of the United States of America, vol. 95, no. 13, pp. 7631-7636, 1998.

[16] E. A. Bristow, P. G. Griffiths, R. M. Andrews, M. A. Johnson, and D. M. Turnbull, "The distribution of mitochondrial activity in relation to optic nerve structure," Archives of Ophthalmology, vol. 120, no. 6, pp. 791-796, 2002.

[17] C. Balaratnasingam, W. H. Morgan, V. Johnstone, S. J. Cringle, and D. Y. Yu, "Heterogeneous distribution of axonal cytoskeleton proteins in the human optic nerve," Investigative Ophthalmology and Visual Science, vol. 50, no. 6, pp. 28242838, 2009.

[18] M. J. Barron, P. Griffiths, D. M. Turnbull, D. Bates, and P. Nichols, "The distributions of mitochondria and sodium channels reflect the specific energy requirements and conduction properties of the human optic nerve head," British Journal of Ophthalmology, vol. 88, no. 2, pp. 286-290, 2004.

[19] V. Hogan, K. White, J. Edgar et al., "Increase in mitochondrial density within axons and supporting cells in response to demyelination in the Plp1 mouse model," Journal of Neuroscience Research, vol. 87, no. 2, pp. 452-459, 2009.

[20] H. Andrews, K. White, C. Thomson et al., "Increased axonal mitochondrial activity as an adaptation to myelin deficiency in the shiverer mouse," Journal of Neuroscience Research, vol. 83, no. 8, pp. 1533-1539, 2006.

[21] S. E. Mutsaers and W. M. Carroll, "Focal accumulation of intra-axonal mitochondria in demyelination of the cat optic nerve," Acta Neuropathologica, vol. 96, no. 2, pp. 139-143, 1998.

[22] W. M. Carroll, A. R. Jennings, and F. L. Mastaglia, "Galactocerebroside antiserum causes demyelination of cat optic nerve," Brain Research, vol. 330, no. 2, pp. 378-381, 1985.

[23] S. Sathornsumetee, D. B. McGavern, D. R. Ure, and M. Rodriguez, "Quantitative ultrastructural analysis of a single spinal cord demyelinated lesion predicts total lesion load, axonal loss, and neurological dysfunction in a murine model of multiple sclerosis," American Journal of Pathology, vol. 157, no. 4, pp. 1365-1376, 2000.

[24] A. Nicot, P. V. Ratnakar, Y. Ron, C. C. Chen, and S. Elkabes, "Regulation of gene expression in experimental autoimmune encephalomyelitis indicates early neuronal dysfunction," Brain, vol. 126, no. 2, pp. 398-412, 2003.

[25] T. Zeis, J. Kinter, E. Herrero-Herranz, R. Weissert, and N. Schaeren-Wiemers, "Gene expression analysis of normal appearing brain tissue in an animal model for multiple sclerosis revealed grey matter alterations, but only minor white matter changes," Journal of Neuroimmunology, vol. 205, no. 12, pp. 10-19, 2008.

[26] X. Qi, A. S. Lewin, L. Sun, W. W. Hauswirth, and J. Guy, "Mitochondrial protein nitration primes neurodegeneration in experimental autoimmune encephalomyelitis," The Journal of Biological Chemistry, vol. 281, no. 42, pp. 31950-31962, 2006. 
[27] S. R. Werner, J. K. Saha, C. L. Broderick et al., "Proteomic analysis of demyelinated and remyelinating brain tissue following dietary cuprizone administration," Journal of Molecular Neuroscience, vol. 42, no. 2, pp. 210-225, 2010.

[28] X. Qi, A. S. Lewin, L. Sun, W. W. Hauswirth, and J. Guy, "Suppression of mitochondrial oxidative stress provides long-term neuroprotection in experimental optic neuritis," Investigative Ophthalmology and Visual Science, vol. 48, no. 2, pp. 681-691, 2007.

[29] R. L. Morris and P. J. Hollenbeck, "Axonal transport of mitochondria along microtubules and F-actin in living vertebrate neurons," Journal of Cell Biology, vol. 131, no. 5, pp. 13151326, 1995.

[30] B. Ferguson, M. K. Matyszak, M. M. Esiri, and V. H. Perry, "Axonal damage in acute multiple sclerosis lesions," Brain, vol. 120, no. 3, pp. 393-399, 1997.

[31] P. S. Yam, T. Takasago, D. Dewar, D. I. Graham, and J. McCulloch, "Amyloid precursor protein accumulates in white matter at the margin of a focal ischaemic lesion," Brain Research, vol. 760, no. 1-2, pp. 150-157, 1997.

[32] A. D. Pilling, D. Horiuchi, C. M. Lively, and W. M. Saxton, "Kinesin-1 and dynein are the primary motors for fast transport of mitochondria in Drosophila motor axons," Molecular Biology of the Cell, vol. 17, no. 4, pp. 2057-2068, 2006.

[33] C. Zhao, J. Takita, Y. Tanaka et al., "Charcot-Marie-Tooth disease type $2 \mathrm{~A}$ caused by mutation in a microtubule motor KIF1B $\beta$," Cell, vol. 105, no. 5, pp. 587-597, 2001.

[34] C. Wang, D. J. Asai, and K. R. Robinson, "Retrograde but not anterograde bead movement in intact axons requires dynein," Journal of Neurobiology, vol. 27, no. 2, pp. 216-226, 1995.

[35] K. J. De vos, A. L. Chapman, M. E. Tennant et al., "Familial amyotrophic lateral sclerosis-linked SOD1 mutants perturb fast axonal transport to reduce axonal mitochondria content," Human Molecular Genetics, vol. 16, no. 22, pp. 2720-2728, 2007.

[36] K. E. Miller and M. P. Sheetz, "Axonal mitochondrial transport and potential are correlated," Journal of Cell Science, vol. 117, no. 13, pp. 2791-2804, 2004.

[37] J. S. Kang, J. H. Tian, P. Y. Pan et al., "Docking of axonal mitochondria by syntaphilin controls their mobility and affects short-term facilitation," Cell, vol. 132, no. 1, pp. 137148, 2008.

[38] A. Olichon, L. Baricault, N. Gas et al., "Loss of OPA1 perturbates the mitochondrial inner membrane structure and integrity, leading to cytochrome c release and apoptosis," The Journal of Biological Chemistry, vol. 278, no. 10, pp. 77437746, 2003.

[39] E. Smirnova, L. Griparic, D. L. Shurland, and A. M. Van der Bliek, "Dynamin-related protein Drp1 is required for mitochondrial division in mammalian cells," Molecular Biology of the Cell, vol. 12, no. 8, pp. 2245-2256, 2001.

[40] H. Chen, S. A. Detmer, A. J. Ewald, E. E. Griffin, S. E. Fraser, and D. C. Chan, "Mitofusins Mfn1 and Mfn2 coordinately regulate mitochondrial fusion and are essential for embryonic development," Journal of Cell Biology, vol. 160, no. 2, pp. 189200, 2003.

[41] M. V. Alavi, S. Bette, S. Schimpf et al., "A splice site mutation in the murine Opal gene features pathology of autosomal dominant optic atrophy," Brain, vol. 130, no. 4, pp. 1029-1042, 2007.

[42] V. J. Davies, A. J. Hollins, M. J. Piechota et al., "Opa1 deficiency in a mouse model of autosomal dominant optic atrophy impairs mitochondrial morphology, optic nerve structure and visual function," Human Molecular Genetics, vol. 16, no. 11, pp. 1307-1318, 2007.

[43] C. Delettre, G. Lenaers, J. M. Griffoin et al., "Nuclear gene OPA1, encoding a mitochondrial dynamin-related protein, is mutated in dominant optic atrophy," Nature Genetics, vol. 26, no. 2, pp. 207-210, 2000.

[44] A. F. Davis and D. A. Clayton, "In situ localization of mitochondrial DNA replication in intact mammalian cells," Journal of Cell Biology, vol. 135, no. 4, pp. 883-893, 1996.

[45] M. Amiri and P. J. Hollenbeck, "Mitochondrial biogenesis in the axons of vertebrate peripheral neurons," Developmental Neurobiology, vol. 68, no. 11, pp. 1348-1361, 2008.

[46] A. Compston and A. Coles, "Multiple sclerosis," The Lancet, vol. 372, no. 9648, pp. 1502-1517, 2008.

[47] C. Bjartmar, J. R. Wujek, and B. D. Trapp, "Axonal loss in the pathology of MS: consequences for understanding the progressive phase of the disease," Journal of the Neurological Sciences, vol. 206, no. 2, pp. 165-171, 2003.

[48] S. M. de Waegh, V. M. Y. Lee, and S. T. Brady, "Local modulation of neurofilament phosphorylation, axonal caliber, and slow axonal transport by myelinating Schwann cells," Cell, vol. 68, no. 3, pp. 451-463, 1992.

[49] B. D. Trapp, J. Peterson, R. M. Ransohoff, R. Rudick, S. Mörk, and L. Bö, "Axonal transection in the lesions of multiple sclerosis," The New England Journal of Medicine, vol. 338, no. 5, pp. 278-285, 1998.

[50] S. M. Gentleman, M. J. Nash, C. J. Sweeting, D. I. Graham, and G. W. Roberts, " $\beta$-Amyloid precursor protein ( $\beta$ APP) as a marker for axonal injury after head injury," Neuroscience Letters, vol. 160, no. 2, pp. 139-144, 1993.

[51] J. T. Povlishock, "Traumatically induced axonal injury: pathogenesis and pathobiological implications," Brain Pathology, vol. 2, no. 1, pp. 1-12, 1992.

[52] D. J. Mahad, I. Ziabreva, G. Campbell et al., "Mitochondrial changes within axons in multiple sclerosis," Brain, vol. 132, no. 5, pp. 1161-1174, 2009.

[53] M. E. Witte, B. Ø. Lars, R. J. Rodenburg et al., "Enhanced number and activity of mitochondria in multiple sclerosis lesions," Journal of Pathology, vol. 219, no. 2, pp. 193-204, 2009.

[54] F. Lu, M. Selak, J. O’Connor et al., “Oxidative damage to mitochondrial DNA and activity of mitochondrial enzymes in chronic active lesions of multiple sclerosis," Journal of the Neurological Sciences, vol. 177, no. 2, pp. 95-103, 2000.

[55] K. J. Smith and H. Lassmann, "The role of nitric oxide in multiple sclerosis," Lancet Neurology, vol. 1, no. 4, pp. 232241, 2002.

[56] L. A. Voloboueva, M. Duan, Y. Ouyang, J. F. Emery, C. Stoy, and R. G. Giffard, "Overexpression of mitochondrial Hsp70/Hsp75 protects astrocytes against ischemic injury in vitro," Journal of Cerebral Blood Flow and Metabolism, vol. 28, no. 5, pp. 1009-1016, 2008.

[57] R. Dutta, J. McDonough, X. Yin et al., "Mitochondrial dysfunction as a cause of axonal degeneration in multiple sclerosis patients," Annals of Neurology, vol. 59, no. 3, pp. 478489, 2006.

[58] H. Neumann, I. M. Medana, J. Bauer, and H. Lassmann, "Cytotoxic T lymphocytes in autoimmune and degenerative CNS diseases," Trends in Neurosciences, vol. 25, no. 6, pp. 313319, 2002.

[59] D. C. Anthony, K. M. Miller, S. Fearn et al., "Matrix metalloproteinase expression in an experimentally-induced 
DTH model of multiple sclerosis in the rat CNS," Journal of Neuroimmunology, vol. 87, no. 1-2, pp. 62-72, 1998.

[60] R. Hohlfeld, "Biotechnological agents for the immunotherapy of multiple sclerosis. Principles, problems and perspectives," Brain, vol. 120, no. 5, pp. 865-914, 1997.

[61] H. Lassmann, “Axonal injury in multiple sclerosis," Journal of Neurology Neurosurgery and Psychiatry, vol. 74, no. 6, pp. 695697, 2003.

[62] A. Kutzelnigg, C. F. Lucchinetti, C. Stadelmann et al., "Cortical demyelination and diffuse white matter injury in multiple sclerosis," Brain, vol. 128, no. 11, pp. 2705-2712, 2005.

[63] P. K. Stys, S. G. Waxman, and B. R. Ransom, " $\mathrm{Na}^{+}-\mathrm{Ca}^{2+}$ exchanger mediates $\mathrm{Ca}^{2+}$ influx during anoxia in mammalian central nervous system white matter," Annals of Neurology, vol. 30, no. 3, pp. 375-380, 1991.

[64] M. J. Craner, B. C. Hains, A. C. Lo, J. A. Black, and S. G. Waxman, "Co-localization of sodium channel $\mathrm{Na}_{\mathrm{v}} 1.6$ and the sodium-calcium exchanger at sites of axonal injury in the spinal cord in EAE," Brain, vol. 127, no. 2, pp. 294-303, 2004.

[65] B. Kornek, M. K. Storch, J. Bauer et al., "Distribution of a calcium channel subunit in dystrophic axons in multiple sclerosis and experimental autoimmune encephalomyelitis," Brain, vol. 124, no. 6, pp. 1114-1124, 2001.

[66] B. D. Trapp and P. K. Stys, "Virtual hypoxia and chronic necrosis of demyelinated axons in multiple sclerosis," The Lancet Neurology, vol. 8, no. 3, pp. 280-291, 2009.

[67] M. Coleman, "Axon degeneration mechanisms: commonality amid diversity," Nature Reviews Neuroscience, vol. 6, no. 11, pp. 889-898, 2005.

[68] D. A. Bechtold, S. J. Miller, A. C. Dawson et al., "Axonal protection achieved in a model of multiple sclerosis using lamotrigine," Journal of Neurology, vol. 253, no. 12, pp. 15421551,2006

[69] D. A. Bechtold, R. Kapoor, and K. J. Smith, "Axonal protection using flecainide in experimental autoimmune encephalomyelitis," Annals of Neurology, vol. 55, no. 5, pp. 607-616, 2004.

[70] A. C. Lo, C. Y. Saab, J. A. Black, and S. G. Waxman, "Phenytoin protects spinal cord axons and preserves axonal conduction and neurological function in a model of neuroinflammation in vivo," Journal of Neurophysiology, vol. 90, no. 5, pp. 3566 3571, 2003.

[71] R. Kapoor, M. Davies, P. A. Blaker, S. M. Hall, and K. J. Smith, "Blockers of sodium and calcium entry protect axons from nitric oxide-mediated degeneration," Annals of Neurology, vol. 53, no. 2, pp. 174-180, 2003.

[72] J. A. Black, S. Liu, M. Carrithers, L. M. Carrithers, and S. G. Waxman, "Exacerbation of experimental autoimmune encephalomyelitis after withdrawal of phenytoin and carbamazepine," Annals of Neurology, vol. 62, no. 1, pp. 21-33, 2007.

[73] R. Kapoor, J. Furby, T. Hayton et al., "Lamotrigine for neuroprotection in secondary progressive multiple sclerosis: a randomised, double-blind, placebo-controlled, parallel-group trial," The Lancet Neurology, vol. 9, no. 7, pp. 681-688, 2010.

[74] M. J. Craner, T. G. Damarjian, S. Liu et al., "Sodium channels contribute to microglia/macrophage activation and function in EAE and MS," Glia, vol. 49, no. 2, pp. 220-229, 2005.

[75] S. G. Waxman, "Axonal dysfunction in chronic multiple sclerosis: meltdown in the membrane," Annals of Neurology, vol. 63 , no. 4 , pp. 411-413, 2008.
[76] J. A. Black, J. Newcombe, B. D. Trapp, and S. G. Waxman, "Sodium channel expression within chronic multiple sclerosis plaques," Journal of Neuropathology and Experimental Neurology, vol. 66, no. 9, pp. 828-837, 2007.

[77] P. K. Stys, S. G. Waxman, and B. R. Ransom, "Ionic mechanisms of anoxic injury in mammalian CNS white matter: role of $\mathrm{Na}^{+}$channels and $\mathrm{Na}^{+}-\mathrm{Ca}^{2+}$ exchanger," Journal of Neuroscience, vol. 12, no. 2, pp. 430-439, 1992.

[78] W. I. McDonald and T. A. Sears, "Effect of demyelination on conduction in the central nervous system," Nature, vol. 221, no. 176, pp. 182-183, 1969.

[79] K. J. Smith and W. I. McDonald, "The pathophysiology of multiple sclerosis: the mechanisms underlying the production of symptoms and the natural history of the disease," Philosophical Transactions of the Royal Society B, vol. 354, no. 1390, pp. 1649-1673, 1999. 


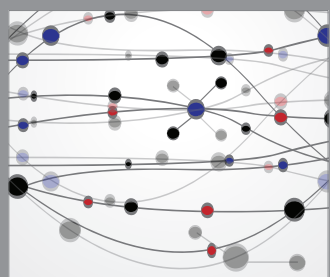

The Scientific World Journal
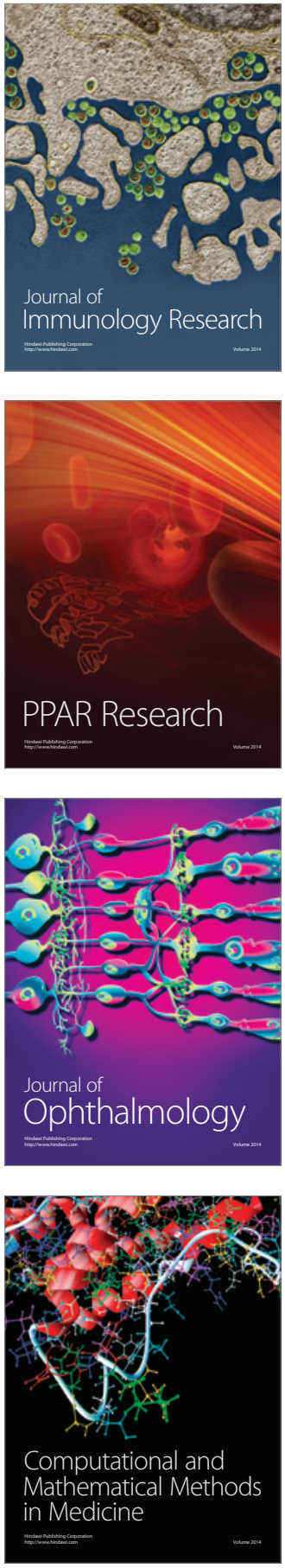

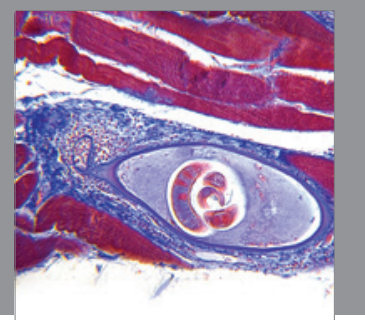

Gastroenterology

Research and Practice
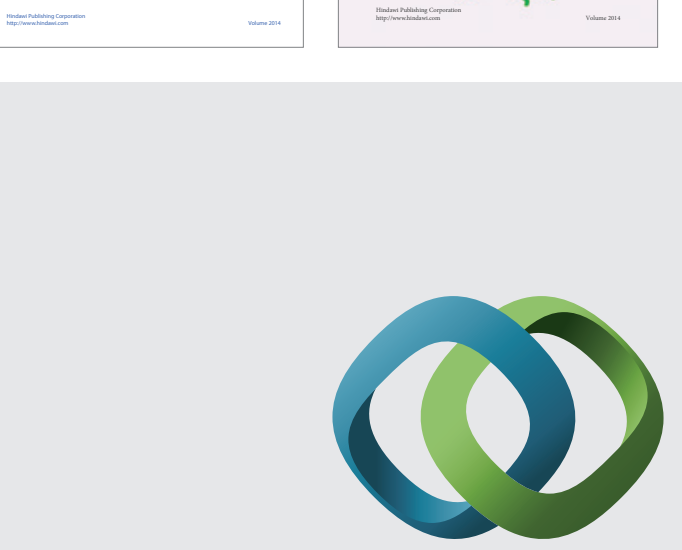

\section{Hindawi}

Submit your manuscripts at

http://www.hindawi.com
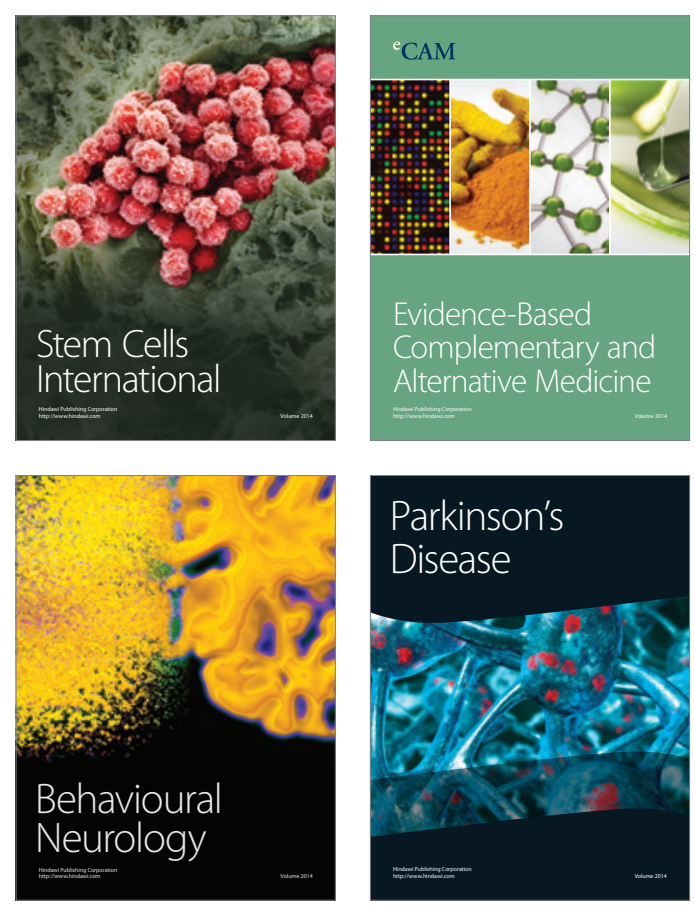

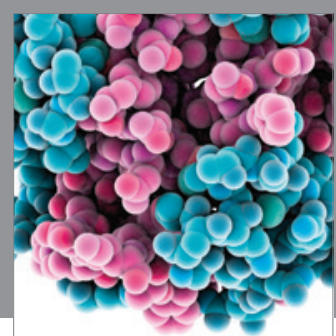

Journal of
Diabetes Research

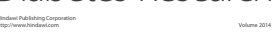

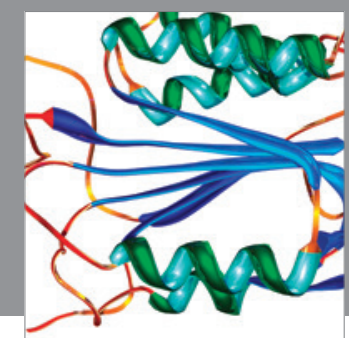

Disease Markers
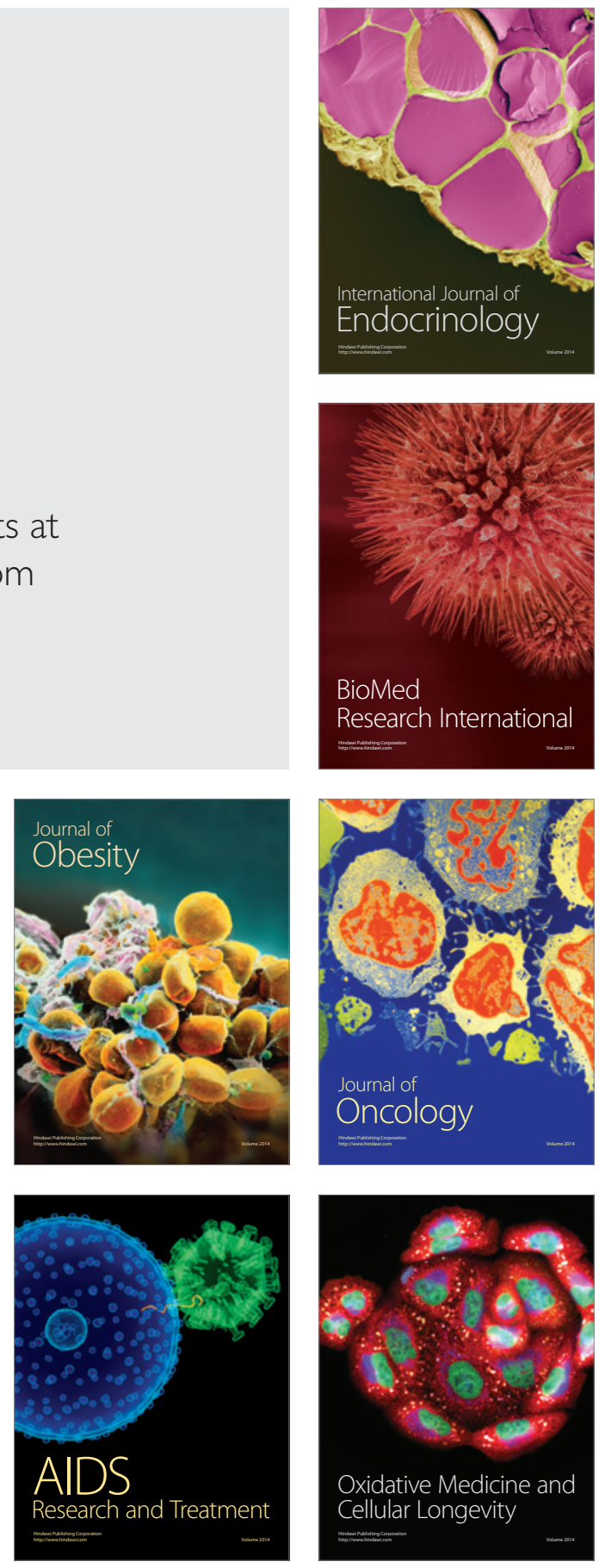